(Koninklijk Nederlands Meteorologisch Instituut, De Bilt)

\title{
A Correction to the Article: "Some Theoretical Considerations with Regard to the Homogeneity-Criteria of Helmert and Abbe ${ }^{1}$ "
}

\author{
By \\ C. Levert
}

R. STEYers was so kind to draw my attention to a mistake I made in my article. Although this mistake does not change my general conclusions, a "complete" correct mathematical treatment of the problem requires correction. First, see pg. 263 , at the bottom, it is obvious that $B=2 A-$ $-2 \underline{F}$ and not $2 \underline{A}-\underline{F}$. Secondly the variance of the quantity $2 A / B$ can be computed in two ways 1 ) as done on pg. 264, using $\varrho(A, B)$ and 2) with the equalities $2 \underline{A} / \underline{B}=1+2 \underline{F} / B$ and $\sigma^{2}(2 A / B)=4 \sigma^{2}(\underline{F} / B)$. Of course I was fully aware (in method 1 ) of the assumption $\varrho(A, \mathrm{~B}) \rightarrow \mathrm{I}$ for $n \rightarrow \infty$ and the approx mative nature of the expressions for $E(\underline{A} / \underline{B})$ and $\sigma^{2}(\underline{A} / \underline{B})$ at the bottom of pg. 264. The second method 2 requires computation of $\sigma^{2}(F / B)$. Approximately $\sigma^{2}(\underline{F} / \underline{B}) \cong \sigma^{2}(F) / E^{2} B$, using $E F=0$. Further $\sigma^{2}(\underline{F})=E E^{2}$, since $E F=0$ and $E^{2} \underline{F}=n \bar{\sigma}^{4}$, since $\bar{E} \varepsilon_{i}{ }^{2} \varepsilon_{i}{ }^{2}+1=\sigma^{4}$, $E \varepsilon_{i} \varepsilon_{i}^{2}{ }_{+1} \varepsilon_{i+2}=0$ and $E \varepsilon_{i} \varepsilon_{i+1} \varepsilon_{i+2} \varepsilon_{i+3}=0$ for $i=1,2, \ldots n$, wich three facts are included in the null-hypothesis: "Each $\varepsilon_{i}$ is independent of each $\varepsilon_{j}$, for $i \neq j$, and each $\varepsilon_{i}$ is distributed normally". Consequently $\sigma^{2}(2 A / B) \cong \sigma^{2}(\underline{F}) / E^{2} B=4 n \sigma^{4} / 4 n^{2} \sigma^{4}=1 / n$, using $E B=2 n \sigma^{2}$ (pg. 264). But this holds only approximately. In this way the value of $\varrho$ ( $\underline{A}, \underline{B}$ ) (which $I$ assumed to be nearly 1 ) follows from $2 \underline{F}=2 \underline{A}-\underline{B}$ and $\sigma^{2}(2 E)=\sigma^{2}(2 A)+\sigma^{2}(B)--2 \varrho(A, B) \sigma(2 A) . \sigma(\underline{B})$, substituting $\sigma^{2}(\underline{A})=2 n \sigma^{4}, \sigma^{2}(\underline{B})=12 n \sigma^{4}$ (pg. 264) and $\sigma^{2}(F)=n \sigma^{4}$ (see above). Hence $\varrho=2 / \sqrt{6}=0.82$.

The consequence of this correction is that $P\left[1-\frac{1}{\sqrt{n}} \leq 2 \frac{\underline{A}}{\underline{B}} \leq 1+\frac{1}{\sqrt{n}}\right]=0.68$ if it is allowed (again an approximation, see pg. 265) to assume that the quantity $2 A(B$ satisfies a normal distribution with mean 1 and standarddeviation $1 / \sqrt{n}$ (under the null-

${ }^{1}$ Archiv Met. Geoph. Biokl. Serie B, 8, 260 (1957) 
hypothesis). It seems more senseful now to consider the limits $1-\frac{2}{\sqrt{n}}$ and $1+\frac{2}{\sqrt{n}}$. Then $P=0.95$ and if the computed $2 A / B$ is situated between these limits we conclude that the null-hypothesis is true (of course this statement has a chance 0.05 of being false). I am lucky to have given a more theoretical base to Putrins' suggestion, made empirically, to change $1 / \sqrt{n}$ into $2 / \sqrt{n}$.

As I said already, although it was necessary to make this correction, my general conclusions rest the same: the interpretation of the results of application of the HELMERT - and ABBE - criteria is fairly difficult. I myself prefer the homogeneity definition, pg. 262 at the top. 